\title{
Schwarz operators of minimal surfaces spanning polygonal boundary curves
}

\section{Journal Article}

Author(s):

Jakob, Ruben

Publication date:

2007

Permanent link:

https://doi.org/10.3929/ethz-b-000422582

Rights / license:

In Copyright - Non-Commercial Use Permitted

Originally published in:

Calculus of Variations and Partial Differential Equations 30(4), https://doi.org/10.1007/s00526-007-0098-5 


\title{
Schwarz operators of minimal surfaces spanning polygonal boundary curves
}

\author{
Ruben Jakob
}

Received: 17 November 2006 / Accepted: 31 December 2006 / Published online: 22 February 2007 (C) Springer-Verlag 2007

\begin{abstract}
This paper examines the Schwarz operator $A$ and its relatives $\dot{A}, \bar{A}$ and $\overline{\dot{A}}$ that are assigned to a minimal surface $X$ which maps consequtive arcs of the boundary of its parameter domain onto the straight lines which are determined by pairs $P_{j}, P_{j+1}$ of two adjacent vertices of some simple closed polygon $\Gamma \subset \mathbb{R}^{3}$. In this case $X$ possesses singularities in those boundary points which are mapped onto the vertices of the polygon $\Gamma$. Nevertheless it is shown that $A$ and its closure $\bar{A}$ have essentially the same properties as the Schwarz operator assigned to a minimal surface which spans a smooth boundary contour. This result is used by the author to prove in [Jakob, Finiteness of the set of solutions of Plateau's problem for polygonal boundary curves. I.H.P. Analyse Non-lineaire (in press)] the finiteness of the number of immersed stable minimal surfaces which span an extreme simple closed polygon $\Gamma$, and in [Jakob, Local boundedness of the set of solutions of Plateau's problem for polygonal boundary curves (in press)] even the local boundedness of this number under sufficiently small perturbations of $\Gamma$.
\end{abstract}

Mathematics Subject Classification (2000) $\quad 49 \mathrm{Q} 05 \cdot 35 \mathrm{P} 15 \cdot 58 \mathrm{E} 12$

\section{Introduction and main results}

This paper is concerned with the Schwarz operator

$$
A \equiv A^{X}:=-\triangle+2 K E
$$

R. Jakob ( $\varangle)$

ETHZ, Rämistr. 101, 8092 Zurich, Switzerland

e-mail: ruben.jakob@math.ethz.ch

Present address:

R. Jakob

Limmatstr. 29, 8005 Zurich, Switzerland 
for a minimal surface $X$ which maps consequtive arcs of the boundary of its parameter domain onto the straight lines that are determined by pairs $P_{j}, P_{j+1}$ of two adjacent vertices of an arbitrarily fixed simple closed polygon $\Gamma \subset \mathbb{R}^{3}$ with $N+3$ vertices. Such a surface is given by a continuous $H^{1,2}$-mapping $X: \bar{B} \longrightarrow \mathbb{R}^{3}$ of the closure of the unit disc $B:=\left\{w=(u, v) \in \mathbb{R}^{2}|| w \mid<1\right\}$ into $\mathbb{R}^{3}$ which is harmonic on $B$, satisfies

$$
\left|X_{u}\right|=\left|X_{v}\right|, \quad\left\langle X_{u}, X_{v}\right\rangle=0 \quad \text { on } B
$$

and meets the boundary conditions $X\left(e^{i \theta}\right) \in \Gamma_{j}$ for $\theta \in\left[\tau_{j}, \tau_{j+1}\right], j=1, \ldots, N+3$, where $\Gamma_{j}$ denotes the line $\left\{P_{j}+t\left(P_{j+1}-P_{j}\right) \mid t \in \mathbb{R}\right\}$ and where the $\tau_{j}$ are consequtive angles in $(0,2 \pi]$. We denote by $\tilde{\mathcal{M}}(\Gamma)$ the set of such surfaces. Furthermore $K$ in $(1)$ is the Gauss curvature of $X$ and $E:=\left|X_{u}\right|^{2}$. For minimal surfaces $X$ bounded by some smooth contour $\Gamma$ the behaviour of $A^{X}$ is well known. The aim of this paper is to show that $A^{X}$ respectively its closure $\overline{A^{X}}$ have essentially the same properties for minimal surfaces $X$ with those "overshooting", piecewise linear boundary values, as explained above. The author is using this result in $[7,8]$ for his proof of the boundedness of the number of immersed stable minimal surfaces spanning a simple closed polygon which is contained in a sufficiently small neighborhood of any fixed extreme simple closed polygon. The difficulty in studying $A^{X}$ for a minimal surface $X$ with overshooting, piecewise linear boundary constraints is caused by the fact that $X$ is "singular" at the boundary points $e^{i \tau_{j}}$ which are mapped onto the corners $P_{j}$ of $\Gamma$. Consequently the perturbing term $K E$ of $A^{X}$ is only of class $L^{p}(B)$ for some $p>1$ on account of estimate (5) below. For some fixed $X \in \tilde{\mathcal{M}}(\Gamma)$ we shall consider $A \equiv A^{X}$ on

$$
\operatorname{Domain}(A):=\left\{\varphi \in C^{2}(B) \cap \stackrel{\circ}{H}^{1,2}(B) \mid A(\varphi) \in L^{2}(B)\right\} .
$$

By $\dot{A}$ and $\dot{\triangle}$ we denote the minimal Schwarz and minimal Laplace operator on the domain $H^{2,2}(B) \cap C_{0}^{2}(B)$, respectively, where we set

$$
C_{0}^{2}(B):=\left\{\varphi \in C^{2}(B) \cap C^{0}(\bar{B})|\varphi|_{\partial B} \equiv 0\right\} .
$$

Furthermore let $\bar{A}, \overline{\dot{A}}$ and $\bar{\triangle}$ denote the $L^{2}(B)$-closures of $A, \dot{A}$ and $\dot{\triangle}$, respectively. Finally we consider the assigned quadratic form

$$
J(\varphi) \equiv J^{X}(\varphi):=\int_{B}|\nabla \varphi|^{2}+2 K E \varphi^{2} \mathrm{~d} w
$$

which is defined for any $\varphi \in \stackrel{\circ}{H}^{1,2}(B)$ due to $K E \in L^{p}(B)$ for some $p>1$. To study the spectra of $A$ and $\bar{A}$ we investigate $J$ on the function space

$$
S \stackrel{\circ}{H}^{1,2}(B):=\left\{\varphi \in \stackrel{\circ}{H}^{1,2}(B) \mid\|\varphi\|_{L^{2}(B)}=1\right\} .
$$

Similarly we denote by $S\left(H^{2,2}(B) \cap \stackrel{H}{H}^{1,2}(B)\right)$ and $S \operatorname{Dom}(A)$ the intersections of the " $L^{2}(B)$-sphere" with the respective function spaces. Then we shall prove

Theorem 1 (i) The spectra of $A$ and $\bar{A}$ coincide. They are discrete and accumulate only at $\infty$; thus their eigenspaces are finite dimensional. Furthermore for their common smallest eigenvalue $\lambda_{\min }:=\lambda_{\min }(A)=\lambda_{\min }(\bar{A})$ we have

$$
\lambda_{\min }=\inf _{S \operatorname{Dom}(A)} J=\inf _{S \dot{H}^{1,2}(B)} J=\inf _{S\left(H^{2,2}(B) \cap H^{1,2}(B)\right)} J .
$$


(ii) For an eigenfunction $\varphi^{*}$ in the eigenspace $\operatorname{ES}_{\lambda_{\min }}(\bar{A})$ there holds $\left|\varphi^{*}\right|>0$ on $B$, whence:

$$
\operatorname{dim} \mathrm{ES}_{\lambda_{\min }}(\bar{A})=\operatorname{dim} \mathrm{ES}_{\lambda_{\min }}(A)=1 .
$$

Especially an eigenfunction $\varphi^{*} \in \mathrm{ES}_{\lambda_{\min }}(A)$ satisfies $\left|\varphi^{*}\right|>0$ on $B$.

To prove this theorem we need some of Heinz' results (see [3,4]) about minimal surfaces with overshooting, piecewise linear boundary values. To this end we need some definitions:

Let $\Gamma$ be some simple closed polygon in $\mathbb{R}^{3}$ with $N+3$ vertices $(N \in \mathbb{N})$

$$
\left(P_{1}, P_{2}, \ldots, P_{N+3}\right),
$$

where we require the pairs of vectors $\left(P_{j+1}-P_{j}, P_{j}-P_{j-1}\right)$ to be linear independent for $j=1, \ldots, N+3$, with $P_{0}:=P_{N+3}$ and $P_{N+4}:=P_{1}$. We consider the open bounded convex set $T$ of $N$-tuples

$$
\left(\tau_{1}, \tau_{2}, \ldots, \tau_{N}\right)=: \tau \in(0, \pi)^{N}
$$

which meet $0<\tau_{1}<\cdots<\tau_{N}<\pi$. Moreover we fix the three angles $\tau_{N+k}:=\frac{\pi}{2}(1+k)$, $k=1,2,3$. Now to any $\tau \in T$ we assign the set of surfaces

$\tilde{\mathcal{U}}(\tau):=\left\{X \in C^{0}\left(\bar{B}, \mathbb{R}^{3}\right) \cap C^{2}\left(B, \mathbb{R}^{3}\right) \mid X\left(e^{i \theta}\right) \in \Gamma_{j} \quad\right.$ for $\left.\theta \in\left[\tau_{j}, \tau_{j+1}\right], 1 \leq j \leq N+3\right\}$,

where $\Gamma_{j}:=\left\{P_{j}+t\left(P_{j+1}-P_{j}\right) \mid t \in \mathbb{R}\right\}, P_{N+4}:=P_{1}$ and $\tau_{N+4}:=\tau_{1}$. On account of Satz 1 in [3] one can define the map

$$
\tilde{\psi}(\tau):=\text { unique minimizer of } \mathcal{D} \text { within } \tilde{\mathcal{U}}(\tau),
$$

where $\mathcal{D}$ denotes Dirichlet's integral. We will also use the notation $X(\cdot, \tau)$ for $\tilde{\psi}(\tau)$. From Satz 1 in [3] and Satz 1 in [4] we quote the following result:

Proposition 1 (i) The surfaces $\tilde{\psi}(\tau)$ are harmonic on $B \quad \forall \tau \in T$.

(ii) The function $\tilde{f}:=\mathcal{D} \circ \tilde{\psi}$ is of class $C^{\omega}(T)$.

(iii) A surface $\tilde{\psi}(\tau)$ is conformally parametrized on $B$, thus a minimal surface in $\tilde{\mathcal{U}}(\tau)$, if and only if $\tau$ is contained in $K(\tilde{f})$, the set of critical points of $\tilde{f}$.

Point (i) of the above theorem and the Courant-Lebesgue Lemma imply (cf. [6, Chap. 4]) that

$$
\begin{aligned}
\tilde{\mathcal{M}}(\Gamma) & \equiv\{\text { set of minimal surfaces on } B\} \cap \bigcup_{\tau \in T} \tilde{\mathcal{U}}(\tau) \cap H^{1,2}\left(B, \mathbb{R}^{3}\right) \\
& =\{X \in \operatorname{image}(\tilde{\psi}) \mid X \text { is also conformally parametrized on } B\} .
\end{aligned}
$$

In the sequel we will only consider points $\tau \in K(\tilde{f})$, thus minimal surfaces $X(\cdot, \tau) \in$ $\tilde{\mathcal{M}}(\Gamma)$, and will denote $A^{\tau}:=-\triangle+2(K E)^{\tau}$ and $J^{\tau}$ for the assigned Schwarz operators and quadratic forms. From [5], (3.3), resp. (34) in [7] we quote that there is some constant const. $(\tau)$, depending on $\tau$ and $\Gamma$ only, such that

$$
\left|(K E)^{\tau}(w)\right| \leq \text { const. }(\tau) \sum_{k=1}^{N+3}\left|w-e^{i \tau_{k}}\right|^{-2+\alpha} \quad \forall w \in B,
$$


for any $\tau \in K(\tilde{f})$ and some fixed $\alpha>0$ that depends only on $\Gamma$. Moreover we are going to use the properties of the Green function (see [6, Proposition 6.1])

$$
\tilde{G}(w, y):=\frac{1}{2 \pi} \log \left(\frac{|1-\bar{w} y|}{|w-y|}\right),
$$

which we consider on $(\bar{B} \times \bar{B}) \backslash \Lambda$ with $\Lambda:=\{(w, y) \in \bar{B} \times \bar{B} \mid w=y\}$. In Proposition 6.2 in [6] the author proved that $\tilde{G}(\cdot, y)$ coincides with the weak $H^{1, s}(B)$-limit (for $s \in(1,2))$ and $L^{p}(B)$-limit [for $\left.p \in(1, \infty)\right] G(\cdot, y)$ of some sequence $G^{\rho_{j}}(\cdot, y)$ of so-called mollified Green functions, for any $y \in B$ (see [6, (5.9), (5.10)]). Moreover we are going to use the assigned potential

$$
\mathcal{G}(\varphi)(w):=\int_{B} \tilde{G}(w, y) \varphi(y) \mathrm{d} y \quad \text { for } w \in \bar{B},
$$

which is well defined for any $\varphi \in L^{r}(B)$, with $r>1$, on account of $\tilde{G}(w, \cdot) \in L^{p}(B)$, $\forall p \in[1, \infty), \forall w \in B$, and $\tilde{G}(w, \cdot) \equiv 0$ on $B, \forall w \in \partial B$, by Proposition 6.1 in [6]. Its most important features are Green's identity for any $\varphi \in H^{2,2}(B) \cap C_{0}^{2}(B)$ and $w \in B$ :

$$
-\varphi(w)=\int_{B} G(w, y) \dot{\Delta} \varphi(y) \mathrm{d} y \equiv \mathcal{G}(\dot{\Delta} \varphi)(w),
$$

and the estimate

$$
\|\mathcal{G}(\varphi)\|_{H^{2,2}(B)} \leq \text { const. }\|\varphi\|_{L^{2}(B)},
$$

for any $\varphi \in L^{2}(B)$. Now on account of the equality $\tilde{G}(\cdot, y) \equiv G(\cdot, y)$ one can combine properties of $\tilde{G}$ with the $L^{p}(B)$-estimate $(5.11)$ in [6] for $G(\cdot, y)$ in order to prove the important assertion (3.11) in [5] (see [6, Proposition 7.1] for the proof), which states that for any $\varphi \in H^{2,2}(B) \cap C_{0}^{2}(B)$ and any $\tau \in K(\tilde{f})$ there holds the estimate

$$
\left|(K E)^{\tau} \varphi(w)\right| \leq c(\tau, \alpha) \sum_{k=1}^{N+3}\left|w-e^{i \tau_{k}}\right|^{-1+\frac{\alpha}{2}}\|\Delta \varphi\|_{L^{2}(B)} \quad \forall w \in B .
$$

By a well-known method (see e.g. [1, p. 108, Satz 2.23]) one proves that $H^{2,2}(B) \cap$ $C_{0}^{2}(B)$ is densely contained in $H^{2,2}(B) \cap \stackrel{\circ}{H}^{1,2}(B)$ w. r. to the $H^{2,2}(B)$-norm. Hence, since the embedding $H^{2,2}(B) \hookrightarrow C^{0}(\bar{B})$ is continuous this implies

Proposition 2 The estimate (9) holds for any $\varphi \in H^{2,2}(B) \cap \stackrel{\circ}{H}^{1,2}(B)$ and for any $\tau \in K(\tilde{f})$.

Now a straight forward reasoning leads to (see [6, Proposition 7.3])

Proposition 3 For any $\varphi \in H^{2,2}(B) \cap \stackrel{H}{H}^{1,2}(B)$ and any $\tau \in K(\tilde{f})$ there holds:

$$
\left\|2(K E)^{\tau} \varphi\right\|_{L^{2}(B)} \leq \frac{1}{2}\|\Delta \varphi\|_{L^{2}(B)}+c\|\varphi\|_{L^{2}(B)},
$$

for some constant $c=c(\tau)$ that only depends on $\tau$.

We will abbreviate $A^{\tau}:=A^{X(\cdot, \tau)}$ and $\dot{A}^{\tau}:=\dot{A}^{X(\cdot, \tau)}$ in the sequel. From Proposition 3 we can derive firstly that $\operatorname{Dom}\left(\dot{A}^{\tau}\right)=H^{2,2}(B) \cap C_{0}^{2}(B)$ is contained 黑 Springer 
in $\operatorname{Dom}\left(A^{\tau}\right)$, thus $\dot{A}^{\tau} \subset A^{\tau}$, and especially that $A^{\tau}$ is densely defined in $L^{2}(B)$, $\forall \tau \in K(\tilde{f})$. Moreover we have

Proposition $4 A^{\tau}$ is symmetric w. r. to $\langle\cdot, \cdot\rangle_{L^{2}(B)}$, i.e., $A^{\tau} \subset\left(A^{\tau}\right)^{*} \forall \tau \in K(\tilde{f})$.

Proof We fix some $\tau \in K(\tilde{f})$. For any $\varphi \in \operatorname{Dom}\left(A^{\tau}\right)$ and $\psi \in C_{c}^{\infty}(B)$ we have $\nabla \varphi \psi \in C_{c}^{1}(B)$. Hence, by the divergence theorem we obtain

$$
\left\langle A^{\tau}(\varphi), \psi\right\rangle_{L^{2}(B)}=\int_{B} \nabla \varphi \cdot \nabla \psi+2(K E)^{\tau} \varphi \psi \mathrm{d} w=: \mathcal{L}^{\tau}(\varphi, \psi) .
$$

Now let $\psi \in \stackrel{\circ}{H}^{1,2}(B)$ be arbitrarily chosen and $\left\{\psi_{j}\right\} \subset C_{c}^{\infty}(B)$ with $\psi_{j} \longrightarrow \psi$ in $\stackrel{\circ}{H}^{1,2}(B)$. By Hölder's inequality and Sobolev's embedding theorem we achieve due to $1-\frac{2}{2}=0>0-\frac{2}{q}, \forall q \in[1, \infty)$ :

$$
\begin{aligned}
\left\|(K E)^{\tau} \varphi\left(\psi_{j}-\psi\right)\right\|_{L^{1}(B)} & \leq\left\|(K E)^{\tau}\right\|_{L^{p^{*}(B)}}\|\varphi\|_{L^{r}(B)}\left\|\psi_{j}-\psi\right\|_{L^{q}(B)} \\
& \leq\left\|(K E)^{\tau}\right\|_{L^{* *}(B)}\|\varphi\|_{L^{r}(B)} \text { const. }(q)\left\|\psi_{j}-\psi\right\|_{H^{1,2}(B)} \longrightarrow 0,
\end{aligned}
$$

for $j \rightarrow \infty$, with $\frac{1}{p^{*}}+\frac{1}{r}+\frac{1}{q}=1$ and $p^{*} \in\left(1, \frac{2}{2-\alpha}\right)$. Hence, recalling that $A^{\tau}(\varphi) \in L^{2}(B)$ we gain (11) in the limit also for $\psi \in \stackrel{H}{H}^{1,2}(B)$, thus especially for any $\psi \in \operatorname{Dom}\left(A^{\tau}\right)$. Together with the symmetry of $\mathcal{L}^{\tau}(\cdot, \cdot)$ this yields for an arbitrary $\varphi \in \operatorname{Dom}\left(A^{\tau}\right)$ :

$$
\left\langle A^{\tau}(\varphi), \psi\right\rangle_{L^{2}(B)}=\mathcal{L}^{\tau}(\varphi, \psi)=\mathcal{L}^{\tau}(\psi, \varphi)=\left\langle\varphi, A^{\tau}(\psi)\right\rangle_{L^{2}(B)}
$$

$\forall \psi \in \operatorname{Dom}\left(A^{\tau}\right)$, which shows indeed $\operatorname{Dom}\left(A^{\tau}\right) \subset \operatorname{Dom}\left(\left(A^{\tau}\right)^{*}\right)$ and $\left(A^{\tau}\right)^{*}(\varphi)=$ $A^{\tau}(\varphi)$, just as asserted.

From this and the symmetry of $\dot{A}^{\tau}$ and $\dot{\Delta}$ on $H^{2,2}(B) \cap C_{0}^{2}(B)$ one can easily derive that $A^{\tau}, \dot{A}^{\tau}$ and $\dot{\triangle}$ are closable in $L^{2}(B), \forall \tau \in K(\tilde{f})$. Now we can prove

Proposition 5 There holds $\operatorname{Dom}(\overline{\dot{\triangle}})=\operatorname{Dom}\left(\overline{\dot{A}}^{\tau}\right)=H^{2,2}(B) \cap \stackrel{\circ}{H}^{1,2}(B) \forall \tau \in K(\tilde{f})$.

Proof We fix some $\tau \in K(\tilde{f})$ arbitrarily and choose some $\varphi \in \operatorname{Dom}(\overline{\dot{\triangle}})$. Thus there is a sequence $\left\{\varphi_{m}\right\} \subset H^{2,2}(B) \cap C_{0}^{2}(B)=\operatorname{Dom}(\dot{\triangle})$ such that

$$
\varphi_{m} \longrightarrow \varphi \text { and } \dot{\triangle} \varphi_{m} \longrightarrow \overline{\dot{\triangle}}(\varphi) \text { in } L^{2}(B) .
$$

By (10) we see that

$$
\left\|2(K E)^{\tau}\left(\varphi_{n}-\varphi_{m}\right)\right\|_{L^{2}(B)} \leq \frac{1}{2}\left\|\dot{\Delta} \varphi_{n}-\dot{\Delta} \varphi_{m}\right\|_{L^{2}(B)}+c\left\|\varphi_{n}-\varphi_{m}\right\|_{L^{2}(B)},
$$

thus that $\left\{2(K E)^{\tau} \varphi_{m}\right\}$ is a Cauchy sequence in $L^{2}(B)$. Now from (13) we can deduce the pointwise convergence

$$
(K E)^{\tau} \varphi_{m_{k}}(w) \longrightarrow(K E)^{\tau} \varphi(w) \text { for a.e. } w \in B,
$$

for some suitable sequence $\left\{m_{k}\right\}$, which shows that $(K E)^{\tau} \varphi_{m} \longrightarrow(K E)^{\tau} \varphi$ in $L^{2}(B)$ and therefore again with (13):

$$
\dot{A}^{\tau}\left(\varphi_{m}\right)=-\dot{\triangle} \varphi_{m}+2(K E)^{\tau} \varphi_{m} \longrightarrow-\overline{\dot{\triangle}}(\varphi)+2(K E)^{\tau} \varphi=\overline{\dot{A}}^{\tau}(\varphi)
$$

in $L^{2}(B)$, which proves that $\varphi \in \operatorname{Dom}\left(\overline{\dot{A}^{\tau}}\right)$. 
Now let some $\varphi \in \operatorname{Dom}\left(\dot{A}^{\tau}\right)$ be given arbitrarily, which means that there exists a sequence $\left\{\varphi_{m}\right\} \subset H^{2,2}(B) \cap C_{0}^{2}(B)$ satisfying

$$
\varphi_{m} \longrightarrow \varphi \text { and } \dot{A}^{\tau}\left(\varphi_{m}\right) \longrightarrow \overline{\dot{A}}^{\tau}(\varphi) \text { in } L^{2}(B) \text {. }
$$

For some arbitrary $\psi \in H^{2,2}(B) \cap C_{0}^{2}(B)$ we have by (10):

$$
\left\|\dot{A}^{\tau}(\psi)\right\|_{L^{2}(B)} \geq\|\dot{\Delta} \psi\|_{L^{2}(B)}-\left\|2(K E)^{\tau} \psi\right\|_{L^{2}(B)} \geq \frac{1}{2}\|\dot{\Delta} \psi\|_{L^{2}(B)}-c\|\psi\|_{L^{2}(B)},
$$

and therefore $\|\dot{\Delta} \psi\|_{L^{2}(B)} \leq 2\left\|\dot{A}^{\tau}(\psi)\right\|_{L^{2}(B)}+2 c\|\psi\|_{L^{2}(B)}$. Combining this with (16) we conclude that $\left\{\dot{\triangle} \varphi_{m}\right\}$ is a Cauchy sequence in $L^{2}(B)$, and therefore also $\left\{2(K E)^{\tau} \varphi_{m}\right\}=\left\{\dot{\Delta} \varphi_{m}+\dot{A}^{\tau}\left(\varphi_{m}\right)\right\}$ due to the second convergence in (16). Now due to the first convergence in (16) we conclude again (15) and thus $(K E)^{\tau} \varphi_{m} \longrightarrow(K E)^{\tau} \varphi$ in $L^{2}(B)$ and therefore again with the second convergence in (16):

$$
\dot{\triangle} \varphi_{m}=-\dot{A}^{\tau}\left(\varphi_{m}\right)+2(K E)^{\tau} \varphi_{m} \longrightarrow-\overline{\dot{A}}^{\tau}(\varphi)+2(K E)^{\tau} \varphi=\overline{\dot{\Delta}} \varphi
$$

in $L^{2}(B)$, i.e., that $\varphi \in \operatorname{Dom}(\overline{\dot{\triangle}})$.

Finally we have to prove that $\operatorname{Dom}(\overline{\dot{\triangle}})=H^{2,2}(B) \cap \stackrel{\circ}{H}^{1,2}(B)$. Firstly let $\varphi \in \operatorname{Dom}(\overline{\dot{\Delta}})$ be chosen arbitrarily, thus there exists a sequence $\left\{\varphi_{m}\right\} \subset H^{2,2}(B) \cap C_{0}^{2}(B)=\operatorname{Dom}(\dot{\triangle})$ satisfying (13). By (7), inequality (8) and (13) we achieve:

$$
\left\|\varphi_{m}\right\|_{H^{2,2}(B)}=\left\|\mathcal{G}\left(\dot{\Delta} \varphi_{m}\right)\right\|_{H^{2,2}(B)} \leq \text { const. }\left\|\dot{\Delta} \varphi_{m}\right\|_{L^{2}(B)} \leq \text { const. }
$$

$\forall m \in \mathbb{N}$. Hence, together with the compactness of the embedding $H^{2,2}(B) \hookrightarrow L^{2}(B)$ and (13) we achieve the existence of a subsequence $\left\{\varphi_{m_{k}}\right\}$ such that $\varphi_{m_{k}} \rightarrow \varphi$ weakly in $H^{2,2}(B)$. This shows indeed $\varphi \in H^{2,2}(B) \cap \stackrel{\circ}{H}^{1,2}(B)$ due to $\stackrel{\circ}{H}^{1,2}(B) \supset \operatorname{Dom}(\dot{\triangle})$. Finally the inclusion $H^{2,2}(B) \cap \stackrel{\circ}{H}^{1,2}(B) \subset \operatorname{Dom}(\overline{\dot{\triangle}})$ follows immediately from the fact that $H^{2,2}(B) \cap C_{0}^{2}(B)$ is densely contained in $H^{2,2}(B) \cap \stackrel{H}{H}^{1,2}(B)$ w. r. to the $H^{2,2}(B)$-norm.

Now we are going to prove the essential self-adjointness of $A^{\tau}$. By means of the continuity of $\mathcal{G}: L^{2}(B) \longrightarrow H^{2,2}(B)$ and (7) one can prove as in [10], p. 59, that $\dot{\Delta}$ is essentially self-adjoint w. r. to $\langle\cdot, \cdot\rangle_{L^{2}(B)}$, i.e., $\overline{\dot{\Delta}}=(\overline{\dot{\triangle}})^{*}$. Together with estimate (10), for $\tau \in K(\tilde{f})$, and the obvious symmetry of $(K E)^{\tau}$ we infer from Theorem 4.4 in $[9$, p. 288]:

Proposition $6 \dot{A}^{\tau}=-\dot{\Delta}+2(K E)^{\tau}$ is essentially self-adjoint w. r. to $\langle\cdot, \cdot\rangle_{L^{2}(B)}$, i.e., $\overline{\dot{A}^{\tau}}=\left(\overline{\dot{A}^{\tau}}\right)^{*}, \forall \tau \in K(\tilde{f})$.

Now combining Proposition 4 with the fact that $\operatorname{Dom}\left(A^{\tau}\right)$ is densely contained in $L^{2}(B)$ w. r. to $\|\cdot\|_{L^{2}(B)}$ we can derive by twice applying Theorem 5.29 in $[9$, p. 168]:

Proposition $7\left(A^{\tau}\right)^{*}$ is densely defined in $L^{2}(B)$ and closed, $\left(A^{\tau}\right)^{* *}=\bar{A}^{\tau}$ and $\left(A^{\tau}\right)^{*}=$ $\overline{\left(A^{\tau}\right)^{*}}=\left(\left(A^{\tau}\right)^{*}\right)^{* *} \forall \tau \in K(\tilde{f})$.

Summarizing we obtain

Proposition $8\left(\dot{A}^{\tau}\right)^{*}=\overline{\dot{A}^{\tau}}=\bar{A}^{\tau}=\left(A^{\tau}\right)^{*}$ are self-adjoint operators with domain $H^{2,2}(B) \cap \stackrel{\circ}{H}^{1,2}(B), \forall \tau \in K(\tilde{f})$.

照 Springer 
Proof We fix some $\tau \in K(\tilde{f})$. Firstly there holds by Proposition 4: $\dot{A}^{\tau} \subset A^{\tau} \subset\left(A^{\tau}\right)^{*}$. Combining this with Propositions 6 and 7 we achieve:

$$
\left(\overline{\dot{A}^{\tau}}\right)^{*}=\overline{\dot{A}^{\tau}} \subset \bar{A}^{\tau} \subset \overline{\left(A^{\tau}\right)^{*}}=\left(\left(A^{\tau}\right)^{*}\right)^{* *}=\left(\left(A^{\tau}\right)^{* *}\right)^{*}=\left(\bar{A}^{\tau}\right)^{*} \subset\left(\overline{\dot{A}^{\tau}}\right)^{*} .
$$

Hence, also noting that $\overline{\left(A^{\tau}\right)^{*}}=\left(A^{\tau}\right)^{*}$ by Proposition 7 , we can conclude that $\overline{\dot{A}^{\tau}}=$ $\bar{A}^{\tau}=\left(A^{\tau}\right)^{*}$ are self-adjoint operators with domain $H^{2,2}(B) \cap \stackrel{\circ}{H}^{1,2}(B)$ by Proposition 6. Furthermore applying Theorem 5.29 in [9, p. 168], to the densely defined and closable operator $\dot{A}^{\tau}$ we obtain that $\left(\dot{A}^{\tau}\right)^{*}$ is densely defined in $L^{2}(B)$, closed, i.e., $\left(\dot{A}^{\tau}\right)^{*}=\overline{\left(\dot{A}^{\tau}\right)^{*}}$, and $\left(\dot{A}^{\tau}\right)^{* *}=\overline{\dot{A}}^{\tau}$. Now applying it to the densely defined and closed operator $\left(\dot{A}^{\tau}\right)^{*}$ again we gain that $\left(\left(\dot{A}^{\tau}\right)^{*}\right)^{* *}=\overline{\left(\dot{A}^{\tau}\right)^{*}}$. Hence, we achieve together with Proposition 6 that

$$
\overline{\dot{A}^{\tau}}=\left(\overline{\dot{A}}^{\tau}\right)^{*}=\left(\left(\dot{A}^{\tau}\right)^{* *}\right)^{*}=\left(\left(\dot{A}^{\tau}\right)^{*}\right)^{* *}=\overline{\left(\dot{A}^{\tau}\right)^{*}}=\left(\dot{A}^{\tau}\right)^{*} .
$$

Now we are going to prove Theorem 1 . As in (11) we will use the bilinear form

$$
\mathcal{L}^{\tau}(\varphi, \psi):=\int_{B} \nabla \varphi \cdot \nabla \psi+2(K E)^{\tau} \varphi \psi \mathrm{d} w,
$$

for $\varphi, \psi \in \stackrel{H}{H}^{1,2}(B)$ assigned to some $\tau \in K(\tilde{f})$, thus especially $J^{\tau}(\varphi) \equiv \mathcal{L}^{\tau}(\varphi, \varphi)$. In the sequel we fix some $\tau \in K(\tilde{f})$, thus some minimal surface $X(\cdot, \tau) \in \tilde{\mathcal{M}}(\Gamma)$, and $p^{*} \in\left(1, \frac{2}{2-\alpha}\right)$ arbitrarily and abbreviate $A:=A^{\tau}, \mathcal{L}:=\mathcal{L}^{\tau}$ and $J:=J^{\tau}$. The final tool of the proof of Theorem 1 is

Proposition 9 There exists some constant $C\left(p^{*}\right)$ such that:

$$
J(\varphi) \geq \frac{1}{2} \int_{B}|\nabla \varphi|^{2} \mathrm{~d} w-C\left(p^{*}\right)\|K E\|_{L^{p^{*}(B)}} \quad \forall \varphi \in S \stackrel{\circ}{H}^{1,2}(B) .
$$

Proof We consider the continuous embeddings $\stackrel{\circ}{H}^{1,2}(B) \hookrightarrow L^{q}(B) \hookrightarrow L^{2}(B)$, for any $q \geq 2$, where the first one is compact due to Sobolev's embedding theorem. Hence, we may apply Ehrling's interpolation lemma, yielding

$$
\|\varphi\|_{L^{q}(B)} \leq \epsilon\|\varphi\|_{\stackrel{H}{H}^{1,2}(B)}+C(q, \epsilon) \quad \forall \varphi \in S \stackrel{\circ}{H}^{1,2}(B),
$$

for any $\epsilon>0$ and any $q \geq 2$, where we used the requirement $\|\varphi\|_{L^{2}(B)}=1$. Hence, together with Hölder's, Cauchy-Schwarz' and Poincaré's inequalities we achieve for any $\epsilon>0$ :

$$
\begin{aligned}
\left\|K E \varphi^{2}\right\|_{L^{1}(B)} & \leq\|K E\|_{L^{p^{*}(B)}}\|\varphi\|_{L^{2 p^{\prime}(B)}}^{2} \leq\|K E\|_{L^{p^{*}(B)}}\left(\epsilon\|\varphi\|_{H^{1,2}(B)}+C\left(p^{\prime}, \epsilon\right)\right)^{2} \\
& \leq\|K E\|_{L^{p^{*}(B)}} 2\left(\epsilon^{2}\left(C_{P}+1\right) \int_{B}|\nabla \varphi|^{2} \mathrm{~d} w+C\left(p^{\prime}, \epsilon\right)^{2}\right),
\end{aligned}
$$

with $\frac{1}{p^{*}}+\frac{1}{p^{\prime}}=1$, and therefore by the definition of $J$ :

$$
J(\varphi) \geq\left(1-4\|K E\|_{L^{p^{*}(B)}}\left(C_{P}+1\right) \epsilon^{2}\right) \int_{B}|\nabla \varphi|^{2} \mathrm{~d} w-4\|K E\|_{L^{p^{*}(B)}} C\left(p^{\prime}, \epsilon\right)^{2},
$$

for any $\varphi \in S H^{1,2}(B)$, which yields our assertion by a suitable choice of $\epsilon$. 
In order to prove Theorem 1 we shall apply Courant's technique for obtaining eigenvalues and eigenfunctions of $A$ by minimizing the quadratic form $J$ on $S H^{1,2}(B)$ with respect to subsidiary conditions. We shall only sketch the necessary steps.

Proof of Theorem 1 Firstly the above proposition guarantees the existence of $\inf _{S H^{1,2}(B)} J$. Hence, we may consider some sequence $\left\{\varphi_{j}\right\} \subset S H^{1,2}(B)$ such that $J\left(\varphi_{j}\right) \searrow \inf _{S H^{1,2}(B)} J$, and again using (18) we conclude together with Poincaré's inequality that $\left\|\varphi_{j}\right\|_{H^{1,2}(B)} \leq$ const. Thus we can extract some subsequence $\left\{\varphi_{j_{k}}\right\}$ such that

$$
\varphi_{j_{k}} \rightarrow \varphi^{*} \quad \text { weakly in } H^{1,2}(B),
$$

for some $\varphi^{*} \in \stackrel{\circ}{H}^{1,2}(B)$. Since this implies $\varphi_{j_{k}} \longrightarrow \varphi^{*}$ in $L^{q}(B)$, for any $q \geq 1$, we infer $\varphi^{*} \in S \stackrel{\circ}{H}^{1,2}(B)$. Furthermore this implies:

$$
\left\|K E\left(\varphi_{j_{k}}^{2}-\left(\varphi^{*}\right)^{2}\right)\right\|_{L^{1}(B)} \leq\|K E\|_{L^{p^{*}(B)}}\left\|\varphi_{j_{k}}^{2}-\left(\varphi^{*}\right)^{2}\right\|_{L^{p^{\prime}(B)}} \longrightarrow 0,
$$

with $\frac{1}{p^{*}}+\frac{1}{p^{\prime}}=1$. Hence, $J$ inherits the weak lower semicontinuity of the Dirichlet integral:

$$
\begin{aligned}
J\left(\varphi^{*}\right) & =\int_{B}\left|\nabla \varphi^{*}\right|^{2}+2(K E)^{\tau}\left(\varphi^{*}\right)^{2} \mathrm{~d} w \\
& \leq \liminf _{k \rightarrow \infty} \int_{B}\left|\nabla \varphi_{j_{k}}\right|^{2} \mathrm{~d} w+2 \lim _{k \rightarrow \infty} \int_{B} K E \varphi_{j_{k}}^{2} \mathrm{~d} w=\liminf _{k \rightarrow \infty} J\left(\varphi_{j_{k}}\right)=\inf _{S \dot{H}^{1,2}(B)} J,
\end{aligned}
$$

thus $J\left(\varphi^{*}\right)=\inf _{S H^{1,2}(B)} J$. Now we construct recursively a filtration of subspaces $\stackrel{\circ}{H}^{1,2}(B)=: U_{1} \supset U_{2} \supset U_{3} \cdots$ of $\stackrel{\circ}{H}^{1,2}(B)$ by

$$
U_{i}:=\left\{\eta \in \stackrel{\circ}{H}^{1,2}(B) \mid\left\langle\eta, \varphi_{j}^{*}\right\rangle_{L^{2}(B)}=0, j=1, \ldots, i-1\right\},
$$

for $i \geq 2$, and $S U_{i}:=U_{i} \cap S \stackrel{\circ}{H}^{1,2}(B)$, where we set $\varphi_{1}^{*}:=\varphi^{*}$ and the $\varphi_{i}^{*} \in S U_{i}$ have to minimize $J$ :

$$
J\left(\varphi_{i}^{*}\right) \stackrel{!}{=} \inf _{S U_{i}} J=: \lambda_{i}
$$

We obtain those minimizers $\varphi_{i}^{*}, i \geq 2$, exactly by the same procedure which yielded $\varphi^{*}$ above since the $U_{i}$ 's are closed w. r. to weak $H^{1,2}(B)$-convergence and non-trivial, otherwise there would hold $\operatorname{Span}\left(\varphi_{1}^{*}, \ldots, \varphi_{i-1}^{*}\right)^{\perp}=\{0\}\left[\perp\right.$ w. r. to $\langle\cdot, \cdot\rangle_{L^{2}(B)}$ in $\left.\stackrel{H}{H}^{1,2}(B)\right]$ which contradicts $\operatorname{dim} H^{1,2}(B)=\infty$ due to the projection theorem. By construction of our filtration the sequence $\left\{\lambda_{i}\right\}$ is increasing. Furthermore $\{\infty\}$ is its only point of accumulation since if there was a bounded subsequence $\left\{\lambda_{i_{k}}\right\}$ then we would conclude by (21), (18) and Poincaré's inequality that $\left\|\varphi_{i_{k}}^{*}\right\|_{H^{1,2}(B)} \leq$ const. $\forall k \in \mathbb{N}$. Hence, since the embedding $H^{1,2}(B) \hookrightarrow L^{2}(B)$ is compact, $\left\{\varphi_{i_{k}}^{*}\right\}$ would possess a Cauchysubsequence w. r. to $\|\cdot\|_{L^{2}(B)}$, which contradicts the fact that

$$
\left\langle\varphi_{i}^{*}-\varphi_{j}^{*}, \varphi_{i}^{*}-\varphi_{j}^{*}\right\rangle_{L^{2}(B)}=\left\|\varphi_{i}^{*}\right\|_{L^{2}(B)}^{2}-2\left\langle\varphi_{i}^{*}, \varphi_{j}^{*}\right\rangle_{L^{2}(B)}+\left\|\varphi_{j}^{*}\right\|_{L^{2}(B)}^{2}=2-2 \delta_{i j}
$$

$\forall i, j \in \mathbb{N}$, by (20) and $\varphi_{i}^{*} \in S U_{i}$. Now we are going to prove that the $\varphi_{i}^{*}$ and $\lambda_{i}$ are indeed eigenfunctions and eigenvalues of $A$ and $\bar{A}$. For some fixed $i$ we consider an arbitrary $\psi \in U_{i}$ and the function

$$
f_{i}(\epsilon):=J\left(\varphi_{i}^{*}+\epsilon \psi\right)-\lambda_{i}\left\|\varphi_{i}^{*}+\epsilon \psi\right\|_{L^{2}(B)}^{2} \text { on }\left[-\epsilon_{0}, \epsilon_{0}\right]
$$


for $\epsilon_{0}>0$ that small such that $\left\|\varphi_{i}^{*}+\epsilon \psi\right\|_{L^{2}(B)}>0 \forall \epsilon \in\left[-\epsilon_{0}, \epsilon_{0}\right]$. Then we obtain for any $\psi \in U_{i}$ and any $i \in \mathbb{N}$, abbreviating $\langle\cdot, \cdot \cdot\rangle:=\langle\cdot, \cdot\rangle_{L^{2}(B)}$ :

$$
0=\left.\frac{\mathrm{d}}{\mathrm{d} \epsilon} f_{i}(\epsilon)\right|_{\epsilon=0}=2\left(\mathcal{L}\left(\varphi_{i}^{*}, \psi\right)-\lambda_{i}\left\langle\varphi_{i}^{*}, \psi\right\rangle\right) .
$$

Next a standard reasoning yields $\mathcal{L}\left(\varphi_{i}^{*}, \psi\right)=\lambda_{i}\left\langle\varphi_{i}^{*}, \psi\right\rangle$ even for any $\psi \in \stackrel{\circ}{H}^{1,2}(B)$, i.e.,

$$
A\left(\varphi_{i}^{*}\right)=\lambda_{i} \varphi_{i}^{*} \quad \text { weakly on } B
$$

$\forall i \in \mathbb{N}$. Now we know that our coefficients $2(K E)^{\tau}-\lambda_{i}$ are of class $C^{\infty}(B)$ for any $\tau \in K(\tilde{f})$ (see [7, (35)]). Thus the $\mathrm{L}^{2}$-regularity theory, Theorem 8.13 in [2], yields that $\varphi_{i}^{*} \in C^{\infty}(B) \forall i \in \mathbb{N}$. Hence, if we test (22) with an arbitrary $\psi \in C_{c}^{\infty}(B)$ and apply the divergence theorem to $\nabla \varphi_{i}^{*} \psi \in C_{c}^{\infty}(B)$, then we obtain:

$$
\left\langle A\left(\varphi_{i}^{*}\right), \psi\right\rangle=\mathcal{L}\left(\varphi_{i}^{*}, \psi\right)=\lambda_{i}\left\langle\varphi_{i}^{*}, \psi\right\rangle .
$$

Thus the fundamental lemma of the calculus of variations yields the Eq. 22 even in the classical sense on $B$. In particular we see that $\varphi_{i}^{*} \in \operatorname{Dom}(A)$, thus indeed the $\varphi_{i}^{*}$ 's and the $\lambda_{i}$ 's are eigenfunctions and eigenvalues of $A$ and therefore also of $\bar{A} \forall i \in \mathbb{N}$. Next a standard reasoning yields $\|\psi\|_{L^{2}(B)}^{2}=\sum_{j=1}^{\infty}\left\langle\varphi_{j}^{*}, \psi\right\rangle^{2}$ for any $\psi \in \stackrel{\circ}{H}^{1,2}(B)$. Now we suppose that $\lambda \notin\left\{\lambda_{i}\right\}$ is a further eigenvalue of $\bar{A}$ and $\phi \in E S_{\lambda}(\bar{A})$ a corresponding eigenfunction. Since $\phi \in H^{2,2}(B) \cap \stackrel{\circ}{H}^{1,2}(B)=\operatorname{Dom}(\bar{A})$ by Theorem 8 we have $\nabla \phi \psi \in \stackrel{\circ}{H}^{1,1}(B)$ for any $\psi \in C_{c}^{\infty}(B)$. Hence, applying the divergence theorem to $\nabla \phi \psi$ we obtain

$$
\mathcal{L}(\phi, \psi)=\langle\bar{A}(\phi), \psi\rangle=\lambda\langle\phi, \psi\rangle,
$$

and we achieve this equality also for any $\psi \in \stackrel{\circ}{H}^{1,2}(B)$ exactly as in the proof of Proposition 4 by approximation. Now testing this weak equation with $\psi:=\varphi_{i}^{*}$ for an arbitrary $i \in \mathbb{N}$ we conclude together with (22):

$$
\lambda\left\langle\phi, \varphi_{i}^{*}\right\rangle=\mathcal{L}\left(\phi, \varphi_{i}^{*}\right)=\mathcal{L}\left(\varphi_{i}^{*}, \phi\right)=\lambda_{i}\left\langle\varphi_{i}^{*}, \phi\right\rangle,
$$

hence, $0=\left(\lambda-\lambda_{i}\right)\left\langle\varphi_{i}^{*}, \phi\right\rangle, \forall i \in \mathbb{N}$, which would imply that all the coordinates $\left\langle\varphi_{i}^{*}, \phi\right\rangle$ of $\phi$ would vanish and therefore $0=\sum_{j=1}^{\infty}\left\langle\varphi_{j}^{*}, \phi\right\rangle^{2}=\|\phi\|_{L^{2}(B)}^{2}$. But $\phi$ is an eigenfunction. Hence, we have proved so far $\left\{\lambda_{i}\right\}=\operatorname{Spec}(\bar{A}) \supset \operatorname{Spec}(A) \supset\left\{\lambda_{i}\right\}$ and therefore also $\left\{\lambda_{i}\right\}=\operatorname{Spec}(A)$. Finally we infer from $\operatorname{Dom}(A) \subset \operatorname{Dom}(\bar{A})=H^{2,2}(B) \cap \stackrel{\circ}{H}^{1,2}(B)$, $\varphi^{*} \in \operatorname{SDom}(A)$ and (19):

$$
\inf _{S \dot{H}^{1,2}(B)} J \leq \inf _{S\left(H^{2,2}(B) \cap \mathscr{H}^{1,2}(B)\right)} J \leq \inf _{S \operatorname{Dom}(A)} J \leq J\left(\varphi^{*}\right)=\inf _{S \dot{H}^{1,2}(B)} J,
$$

which together with $\inf _{S H^{1,2}(B)} J=\lambda_{1}=\lambda_{\min }(A)=\lambda_{\min }(\bar{A})$ completes also the proof of (3). The second part of the theorem now follows along usual lines by employing Harnack's inequality. Let $\varphi^{*} \in E S_{\lambda_{\text {min }}}(\bar{A}) \subset H^{2,2}(B) \cap \stackrel{\circ}{H}^{1,2}(B)$ with $\left\|\varphi^{*}\right\|_{L^{2}(B)}=1$ be given arbitrarily. We assume the existence of some point $w_{0} \in B$ with $\varphi^{*}\left(w_{0}\right)=0$. Firstly we note that $\left|\varphi^{*}\right| \in \stackrel{\circ}{H}^{1,2}(B)$ and that $\int_{B}|\nabla| \varphi^{*}||^{2} \mathrm{~d} w=\int_{B}\left|\nabla \varphi^{*}\right|^{2} \mathrm{~d} w$. Moreover applying (23) to $\phi:=\varphi^{*}$ and $\psi:=\varphi^{*}$ we obtain by (3):

$$
J\left(\left|\varphi^{*}\right|\right)=J\left(\varphi^{*}\right)=\left\langle\bar{A}\left(\varphi^{*}\right), \varphi^{*}\right\rangle_{L^{2}(B)}=\lambda_{\min }\left\langle\varphi^{*}, \varphi^{*}\right\rangle_{L^{2}(B)}=\lambda_{\min }=\inf _{S \dot{H}^{1,2}(B)} J .
$$


Hence, exactly as we achieved (22) we conclude now due to $\left|\varphi^{*}\right| \in \stackrel{\circ}{H}^{1,2}(B)$ :

$$
A\left(\left|\varphi^{*}\right|\right)=\lambda_{\min }\left|\varphi^{*}\right| \quad \text { weakly on } B .
$$

Now we may apply Harnack's inequality, Theorem 8.20 in [2], to $\left|\varphi^{*}\right| \geq 0$ on any disc $B_{4 R}\left(w_{0}\right) \subset \subset B$, yielding $\sup _{B_{R}\left(w_{0}\right)}\left|\varphi^{*}\right| \leq$ const. $\inf _{B_{R}\left(w_{0}\right)}\left|\varphi^{*}\right|$. Hence, from $\varphi^{*}\left(w_{0}\right)=$ 0 we can conclude now that $\varphi^{*} \equiv 0$ on $B_{R}\left(w_{0}\right)$ and thus that $\varphi^{*} \equiv 0$ on $B$ by a successive use of Harnack's inequality, which contradicts our assumption $\left\|\varphi^{*}\right\|_{L^{2}(B)}=1$. Thus we have proved indeed for an arbitrary eigenfunction $\varphi^{*} \in E S_{\lambda_{\min }}(\bar{A})$ that $\varphi^{*}>0$ or $<0$ on $B$. Now we assume that $\operatorname{dim} E S_{\lambda_{\min }}(\bar{A})>1$. On account of the projection theorem we could choose two $\mathrm{L}^{2}(B)$-orthogonal eigenfunctions $\varphi^{*}, \bar{\varphi}^{*}$ in $\operatorname{ES}_{\lambda_{\text {min }}}(\bar{A})$, i.e., with $\left\langle\varphi^{*}, \bar{\varphi}^{*}\right\rangle_{L^{2}(B)}=0$, in contradiction to $\left\langle\varphi^{*}, \bar{\varphi}^{*}\right\rangle_{L^{2}(B)}>0$ or $<0$. As we have $\{0\} \neq E S_{\lambda_{\min }}(A) \subset E S_{\lambda_{\min }}(\bar{A})$ we arrive at $(4)$.

Acknowledgments The author was supported by a research stipend of the Deutsche Forschungsgemeinschaft and would like to thank Prof. Ph.D. Tromba and Prof. Dr. Dierkes for their interest and hospitality and Prof. Dr. Hildebrandt for his support.

\section{References}

1. Alt, H.W.: Lineare funktional analysis 3. Auflage. Springer, Berlin (1999)

2. Gilbarg, D., Trudinger, N.S.: Elliptic partial differential equations of second order, 3rd edn. Classics Math. Springer, Berlin (1998)

3. Heinz, E.: Über die analytische Abhängigkeit der Lösungen eines linearen elliptischen Randwertproblems von den Parametern. Nachr. Akad. Wiss. in Göttingen, II. Math.-Phys. Kl. Jahrgang, 1-20 (1979)

4. Heinz, E.: Zum Marx-Shiffmanschen Variationsproblem. J. Reine U. Angew. Math. 344, 196$200(1983)$

5. Heinz, E.: Minimalflächen mit polygonalem Rand. Math. Zeitschr. 183, 547-564 (1983)

6. Jakob, R.: Finiteness of the set of solutions of Plateau's problem with polygonal boundary curves. Bonner Math. Schriften 379, 1-95 (2006)

7. Jakob, R.: Finiteness of the set of solutions of Plateau's problem for polygonal boundary curves. I.H.P. Analyse Non-lineaire (in press). doi: 10.1016/j.anihpc.2006.10.003

8. Jakob, R.: Local boundedness of the set of solutions of Plateau's problem for polygonal boundary curves. Ann Glob Anal Geom (2007) (submitted)

9. Kato, T.: Perturbation theory for linear operators. Springer, Berlin (1976)

10. Wienholtz, E.: Halbbeschränkte partielle Differentialoperatoren zweiter Ordnung vom elliptischen Typus. Math. Annalen 135, 50-80 (1958) 